\title{
ON THE INDECOMPOSABILITY OF COMPACT CONVEX SETS
}

\author{
MICHAEL EDELSTEIN \\ (Communicated by Palle E. T. Jorgensen)
}

\begin{abstract}
Let $C$ be a compact convex set in a Hausdorff, locally convex linear topological space $X$ and let $\mathscr{F}$ be the family of affine homeomorphisms of $X$ onto itself. It is proved that $C$ is indecomposable under $\mathscr{F}$; i.e. if $C=A \cup B$ and $B=F[A]$, for some $F \in \mathscr{F}$, then $A \cap B \neq \varnothing$.
\end{abstract}

\section{INTRODUCTION}

A set $S$ in a metric space $X$ is said to be decomposable, under the family of isometries of $X$ onto itself, if it is the disjoint union of two isometric subsets. In [1] it was shown that weakly compact convex subsets of a Banach space are not decomposable under the affine isometries of the space onto itself.

It is the purpose of this paper to prove the stronger

Theorem. Let $C$ be a compact convex subset of a Hausdorff, locally convex linear topological space $X$. Then $C$ is not decomposable under the family of affine homeomorphisms of $X$ onto itself.

(For a brief history of closely related results see [1]; for general background material see the comprehensive survey [2] by Stan Wagon.)

Proof of the theorem. Let $\mathscr{F}$ be the family of affine homeomorphisms of $X$ onto itself. Let $F \in \mathscr{F}$ and suppose that $C=A \cup B$ and $B=F[A]$. We have to prove that $A \cap B \neq \varnothing$. Let $0(x)=\left\{F^{n}(x): n \geq 0\right\}$. Two mutually exclusive cases arise:

(i) there is an $x \in C$ such that $0(x) \subset C$; and

(ii) there is no such $x$.

In case $(\mathrm{i})$, since $F$ is both affine and continuous, $\overline{c o}(0(x))$ is mapped into itself, and by known theorems, e.g., one by Tychonov [1, p. 107], a $\xi \in C$ exists such that $F(\xi)=\xi$. It then readily follows that $\xi$ is common to $A$ and $B$. In case (ii), to any $x \in C$ there is a positive integer $n$ such that $F^{n}(x) \in X \backslash C$. Let $\rho: C \rightarrow \mathbb{N}$ be the mapping sending $x \in C$ to the least $n \in \mathbb{N}$ with the property that $F^{n}(x) \in X \backslash C$. As can be readily seen, $\rho$ is bounded. (If not, we may for each $n \in \mathbb{N}$ choose an $x$ with $\rho(x) \geq n$. The set $D$ so obtained has at least one accumulation point $\bar{x}$. If $\rho(\bar{x})=N$ then

Received by the editors October 3, 1990.

1991 Mathematics Subject Classification. Primary 47B99, 52A07. 
$F^{N}(\bar{x}) \in X \backslash C$. However, $F^{-N}[X \backslash C]$ is a neighbourhood of $\bar{x}$ that contains some $y$ with $\rho(y) \geq N+1$; so $\rho(y) \notin X \backslash C$, a contradiction.) Let $N=$ $\sup \rho[C]$ and let $M=\{x \in C: \rho(x)=N\}$. Clearly $M \neq \varnothing$ and if $x \in M$, then $F^{-1}(x) \in X \backslash C$. (Otherwise, with $u=F^{-1}(x), u, F(u), \ldots, F^{n}(u)$ are all members of $C$, and $\rho(u) \geq N+1$.) This fact, in turn, implies that $x \in A$ as $B$ is the one-one image of $A$.

Let $P(x)$ be the polygonal arc joining consecutive members of $F^{-1}(x), x$, $\ldots, F^{N}(x)$ with the closed line segments $\left[F^{k-1}(x), F^{k}(x)\right]$ for $0 \leq k \leq N$. If $x \in M, P(x)$ has the special properties:

(1) $\left[F^{-1}(x), x\right] \cap B=\varnothing$ and

(2) $\left[F^{N-1}(x), F^{N}(x)\right] \cap A=\varnothing$.

Indeed, if $u=\lambda x+(1-\lambda) F^{-1}(x) \in B$ for some $\lambda, 0<\lambda<1$, then $F^{-1}(u) \in$ $A \subset C$ and, more generally, $F^{k}(u)=\lambda F^{k}(x)+(1-\lambda) F^{k-1}(x) \in C$ for $0 \leq$ $k \leq N-1$. Hence $u \in B$ implies that $\left\{F^{-1}(u), u, \ldots, F^{N-1}(u)\right\} \subset C$ and, therefore, $\rho\left(F^{-1}(u)\right) \geq N+1$ against the maximality property of $M$. An analogous argument proves $(2)$.

It follows that $\left[F^{-1}(x), x\right] \cap C=[y, x]$, where $[y, x]$ is a closed line segment in $A$ with end points $y, x$. Similarly, $\left[F^{N-1}(x), F^{N}(x)\right] \cap C=$ $\left[F^{N-1}(x), z\right]$ is a closed line segment in $B$ with end points $F^{N-1}(x), z$. The polygonal arc $P^{*} \subset P(x)$ with end points $y, z$ is, thus, a subset of $C$ and $A \cap P^{*}$ is mapped by $F$ onto $B \cap P^{*}$. We claim that $P^{*}$ is a simple arc; i.e., two line segments

$$
\left[F^{m}(x), F^{m+1}(x)\right] \cap C \text { and }\left[F^{n}(x), F^{n+1}(x)\right] \cap C,
$$

with $n \geq m+2$, are disjoint. To prove this claim observe, first, that if $u \in$ $[x, F(x)]$ then $\rho(u) \geq N-1$ since $\left[F^{N-1}(x), F^{N-1}(x)\right] \subset C$. Suppose now that $\left[F^{m}(x), F^{m+1}(x)\right] \cap C$ intersects $\left[F^{n}(x), F^{n+1}(x)\right]$. (Clearly (1) and (2) imply that either $m \neq-1$ or $n \neq N-1$; and it readily follows that $n-m \leq$ $N-1$.) Applying $F^{-n}$ to both segments there must be a point $v$ that is common to $\left[F^{m-n}(x), F^{m+1-n}(x)\right]$ and $[x, F(x)]$. Further, $v=F^{m-n}(u)$ for some $u \in[x, F(x)]$ and therefore

$$
\rho(v)=\rho(u)+n-m \geq N-1+2=N+1,
$$

contradicting the maximality property of $N$.

We conclude that $P^{*}$ is a homeomorph of $[0,1] \subset \mathbb{R}$ in the usual topology. Hence the natural order of $[0,1]$ induces a corresponding order on $P^{*}$, with 0 corresponding to $y$ and 1 to $z$. Thus it makes sense to refer, for example, to a left-closed, right-open $[\cdot, \cdot)$ subarc. Let $K_{0}$ be the subarc of $P^{*}$ extending from $y$ to $F(y)$ (with $F(y)$ removed, i.e., $K_{0}=[y, x] \cup[x, F(y))$ ). As noted above $[y, x] \subset A$; and similarly, $[x, F(y))$, as the image of $\left[F^{-1}(x), y\right) \subset$ $X \backslash C$, is a subset of $A$. Hence $K_{0} \subset A$ and $x$ is in its relative interior. Thus $A \cap B=\varnothing$ implies that $K_{0} \cap B=\varnothing$. Let $K_{1}=F\left[K_{0}\right]$. Then $K_{1} \cap K_{0}=\varnothing$ and $K_{1}$ is of the form $[F(y), F(x)] \cup\left[F(x), F^{2}(y)\right)$, i.e., the left-closed, rightopen subarc $\left[F(y), F^{2}(y)\right)$; and $F(x)$ is in its relative interior. This type of configuration is preserved under $F$ and terminates in $K_{N-1} \subset B$. Thus $P^{*} \cap A=K_{0} \cup K_{2} \cup \cdots \cup K_{N-2}$, while its image under $F$ is $K_{1} \cup K_{3} \cup \cdots \cup K_{N-1}$. Hence the assumption that $A \cap B=\varnothing$ leads to the absurd conclusion that $P^{*}$ is the finite disjoint union of left-closed, right-open subarcs $K_{i}, 0 \leq i \leq N-1$. Hence $A \cap B \neq \varnothing$, completing the proof of the theorem. 


\section{REFERENCES}

1. Mahlon M. Day, Normed linear spaces, Ergeb. Math. Grenzgeb. (3) 21 (1973).

2. M. Edelstein, K. Johnson, and A. C. Thompson, On the isometric dissection problem for convex sets, Studia Sci. Math. Hungar. (to appear).

3. Stan Wagon, The Banach-Tarski paradox, Cambridge Univ. Press, Cambridge, 1986.

Department of Mathematics, Dalhousie University, Halifax, Nova Scotia, B3H 3J5 CANADA 